\title{
TEXTURES IN CERAMICS
}

\author{
H. J. BUNGE \\ Department of Physical Metallurgy, Technical University of Clausthal, Germany
}

(Received 6 February 1995)

The texture is defined as the orientation distribution of the crystallites in a polycrystalline material. This definition is the same for all kinds of materials. Also the motivation for texture studies is the same in all materials i.e. the formation of textures by anisotropic solid state process and the influence of textures on anisotropic physical or technological properties. Nevertheless, there are some differences in detail between texture analysis in metals and ceramics. These are mainly due to the more complex nature of diffraction diagrams of ceramics as compared to those of the basic metals. The aggravations of texture analysis in ceramics can, however, be overcome by modern experimental techniques e.g. by using a position sensitive detector. Texture analysis in ceramics has also been carried out by microscopical methods. The so obtained "shape texture" is related to the crystallographic texture by a "habitus" function. The particular conditions for texture analysis in cermics are very similar to those encountered in geological materials.

KEY WORDS: Peak-rich diffraction diagrams, low symmetry, microabsorption, particle shape texture, habitus function.

\section{TEXTURES IN POLYCRYSTALLINE MATERIALS}

The texture, as we usually define it, is the orientation distribution of the crystallites in a polycrystalline material. This definition is completely independent of the nature of the crystallites, i.e. whether they are metallic, ceramic, polymeric, geologic or even biologic. Hence, from the very definition of texture there is no need to distinguish textures in ceramics from textures in all other polycrystalline materials.

Also the motivation why we are interested in textures is very much the same in all classes of materials. The two main aspects are illustrated in Figure 1.

- The texture influences all macroscopic properties of a material which are crystallographically anisotropic.

- Textures are formed or modified by all kinds of orientation dependent physical processes which have taken place in the material during its history.

The properties listed in Figure 1 are general features of all solid state materials of all classes. The same holds for the processes. Hence, also from this point of view there is no need to consider textures in ceramics apart from textures in all other classes of materials. A distinction between the different classes of materials may eventually be made according to the emphasis put on a particular property or to the role which a particular process plays in the production of that material. For instance, plastic deformation by dislocation gilde is one of the most important processes for texture formation in metals whereas it has - up to now - virtually not played any important role in the production of ceramics. On the other hand, rigid rotation of particles is 
one of the most important processes during green-forming of ceramics whereas its contribution to texture formation in metals is of only minor importance.

The situation is, however, rapidly changing. Plastic deformation by dislocation glide is also being considered in ceramics although at different temperature ranges, and particle rotation must be taken into consideration in powder metallurgy.

If we look into the literature on textures of materials we find several reviews and monographs which are particularly dedicated to metals such as those by Underwood (1961), Wassermann and Grewen (1962), Kudrjavzev (1965), Coulomb (1972), Hatherly and Hutchinson (1979), but up to now, nothing comparable exists on textures in ceramics. The same holds, in principle, if we consider the proceedings of the ICOTOM conferences. Although they cover, per definition, the whole range of materials, more than $95 \%$ of all papers in these proceedings, in fact, deal with metals. (e.g. Proceedings of ICOTOM-10 (1994)).

This overwhelming emphasis on metals in texture analysis compared to other classes of materials must have particular reasons.

Texture studies in a particular material will be undertaken under the following aspects:

1. There must be a particular interest to study textures in that particular material.

2. The methodical techniques for texture analysis in this class of material must be available.

An interest in texture analysis in a material is often established from a technological point of view, considering the properties listed in Figure 1. The texture can influence a macroscopic property in a variation range given by the crystallographic anisotropy of this property. As soon as this variation range becomes comparable with the allowed variation range of this property according to the actual technological standards then texture studies with the purpose of texture control in the production process become necessary. Since the technological standards are ever increasing this is bound to happen sooner or later for any property in any material.

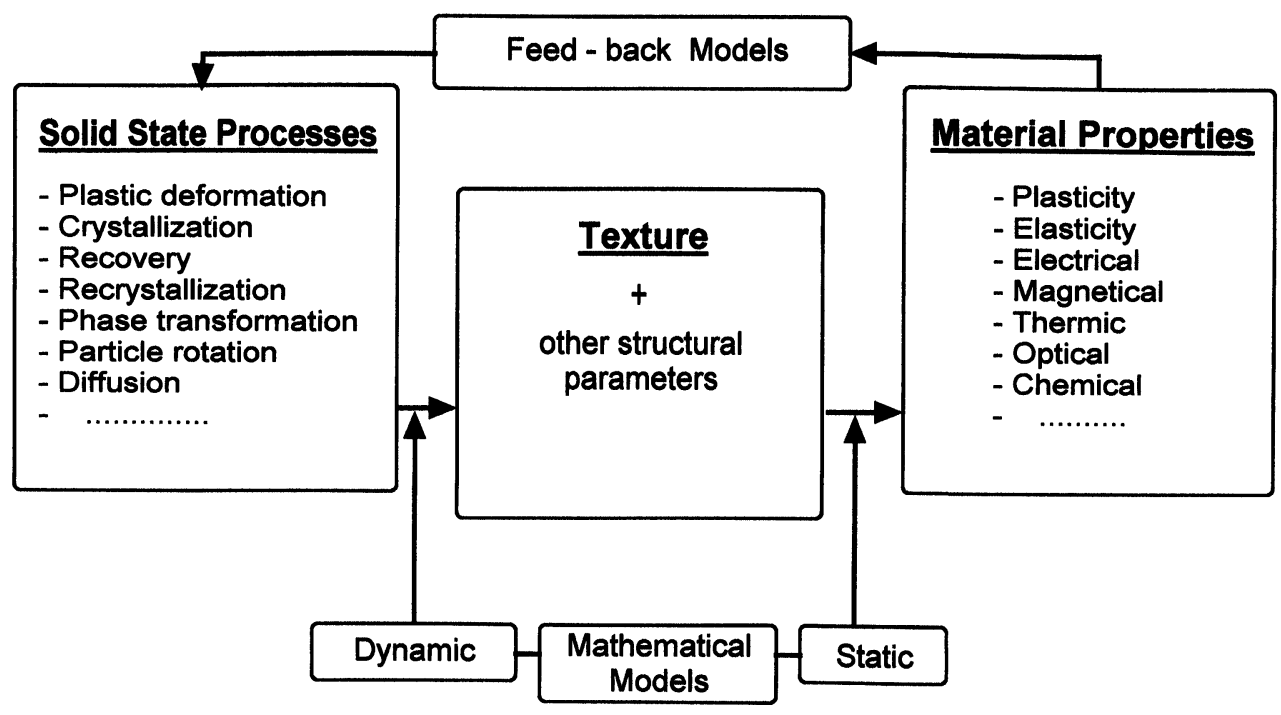

Figure 1 Texture forming processes and the influence of textures on materials properties. 
The second aspect is the feasibility of quantitative texture analysis. In the conventional method of pole figure measurement a wide detector entrance slit is used in order that the complete profile of a diffraction peak, even at high sample tilt angles, falls into it. This method fails for peak-rich diffraction diagrams because of peak overlap.

Both mentioned aspects, the interest in texture analysis and its feasibility must be considered as distinguishing factors between metals and ceramics. In the classical ceramics the technological standards did not realy require a quantitative texture control. Also, these ceramics based on clay minerals have complex crystal structures with peakrich diffraction spectra which do not allow quantitative texture analysis with the conventional techniques. The situation was quite different in metals. They have simple crystal structures allowing quantitative texture analysis with the conventional technique and there was a strong technological motivation for texture studies in metals with increasing technological standards (See e.g. Wassermann and Grewen (1962)).

Both these distinctions between metals and ceramics are, however, no longer valid. In so-called "High-Tech" ceramics, the technological standards are narrower than the variation range introduced by uncontrolled textures variations. Furthermore, several new experimental techniques of texture analysis have recently been developed which allow quantitative texture studies even in materials with peak-rich diffraction diagrams. Because of these reasons the interest in textures in ceramics is rapidly increasing (Bunge (1991), Bowman (1994)).

\section{PARTICULAR CONDITIONS IN CERAMICS}

The term "Ceramics" is usually applied to non-metalic, anorganic materials which are technologically produced (thus excluding natural geological materials belonging to the same class of substances, although these are, in general, the raw materials for the production of ceramics).

In a narrower sense it is often understood that ceramics are produced by a twostep procedure consisting of "green-forming" and "sintering". This definition would exclude, however, such important classes of materials as glass-ceramics or production technologies such as a vapour deposition or melt-texturing of HTc superconductors.

In the present context, we shall include these types of materials and production technologies into our considerations. Most of these considerations then also apply to natural geological materials. Textures in geological materials have been attracting interest continuously over a long period of time. In fact, textures in minerals were already studied by microscopic methods before X-ray diffraction was available (See e.g. Sander (1930)). Surveys on textures in geological materials are contained in the proceedings of conferences on these topics (e.g. Wenk (1985), Bunge et al. (1994)). When comparing metals with ceramics the following structural features of ceramics must be taken into consideration:

- Lower crystal symmetries

- Bigger unit cells

- Lower peak intensities

- Higher background scattering

- Amorphous glass phases

- Pores and microcracks

- Crystallographic shapes of crystals (plates, needles) 
- Variation of the crystal structure (polytypes, occupancy of lattice sites)

- Multiphase composition

This gives rise to the following particluar problems which must be considered in texture analysis of ceramics.

\section{Peak-rich diffraction diagrams}

Diffraction diagrams of ceramics often contain much more peaks than diagrams of metals. This is due to lower crystal symmetries and/or bigger unit cells. This effect may be aggravated in multiphase materials. Figure $2 a$, for instance, is the diffraction diagram of a lithium silicate glass-ceramic, the diffraction peaks of which are very close to each other even in the untilted sample orientation $\alpha=0$. Sample tilt in the range $0 \leq \alpha \leq \alpha_{\max }$ (e.g. $\alpha_{\max }=80^{\circ}$ ) leads to peak broadening as is shown in Figure $2 \mathrm{~b}$. In the conventional method of pole figure measurement the detector entrance slit must be wide enough to accept the integral intensity of the whole diffraction peak. It is seen that then the peaks in Figure $2 b$ cannot be seperated. Hence, texture analysis with the conventional method is thus virtually impossible in a material as that of Figure 2.

It has, however, been shown (Wcislak at al. (1993)) that the peaks of Figure $2 b$ can be separated using a position sensitive detector and mathematical spectrum deconvolution. This way, the pole figures of Figure $2 \mathrm{c}$ were obtained (Wcislak and Nauer-Gerhardt (1994)).

\section{Low-intensity peaks}

As a consequence of the more complex crystal structure, some important diffraction peaks may have a low relative (and absolute) intensity. A prominent example is the (0006)-peak in $\alpha-\mathrm{Al}_{2} \mathrm{O}_{3}$, Figure 3a. Its relative intensity is $<1 \%$ of the strongest peak. Conventional pole figure measurement, in this case, is very difficult. Using the PSDtechnique, pole figures of weak peaks can, however, be measured with sufficient accuracy and with "affordable" measuring times as is shown in Figure 3c.

\section{Camouflaged textures}

In $\mathrm{Al}_{2} \mathrm{O}_{3}$ substrates a basal texture is often observed. In this case the weak basal peak (0006) exhibits the highest pole densities, Figure 3c. Since its intensity is so weak, however, often the pole figures of the high-intensity peaks Figure $3 \mathrm{~b}$ are being measured. According to the particular texture type, i.e. a basal texture, the pole figures of these peaks show only low values of the pole density. This has been called a "camouflaged" texture, because judging the texture qualitatively by the pole figures of Figure $3 \mathrm{~b}$ would lead to the conclusion that the texture is weak whereas, in fact, it is strong.

\section{Low-symmetry ODF-analysis}

ODF-analysis in low-symmetry materials requires the availability of a corresponding ODF-analysis program. Programs dealing with symmetries down to orthorhombic are now available as routine program systems (e.g. Park et al. (1995)). Program systems for lower symmetry e.g. monoclinic have also been developed (Dahms et al. (1994)). Low symmetry ODF-analysis often implies further aggravations: 

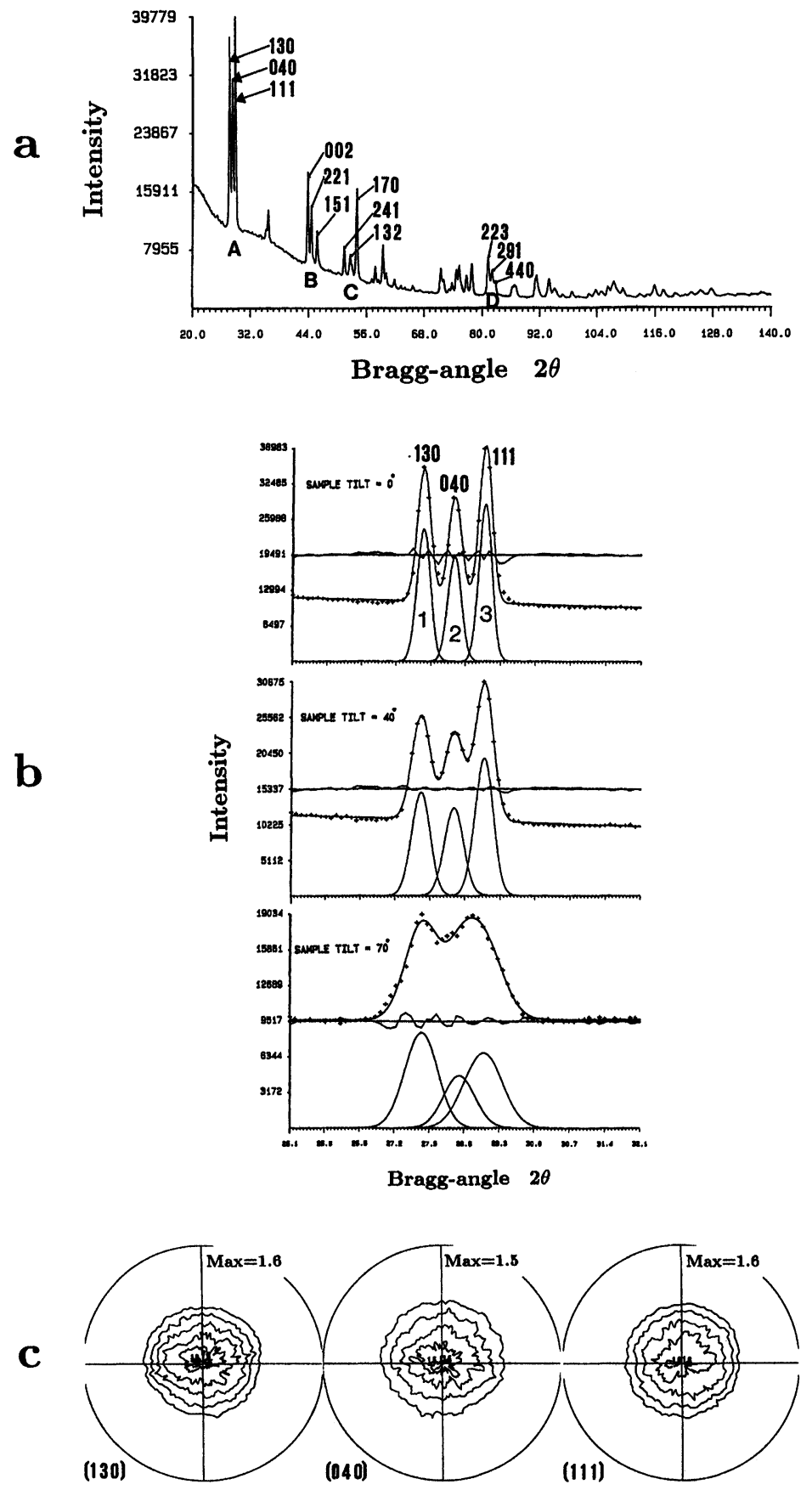

Figure 2 Texture analysis in a lithium silicate glass-ceramic a) Spectrum at zero tilt $\alpha=0$. b) Enlarged plot of the spectrum at different tilt angles. c) Pole figures of the three peaks of part $b$. 

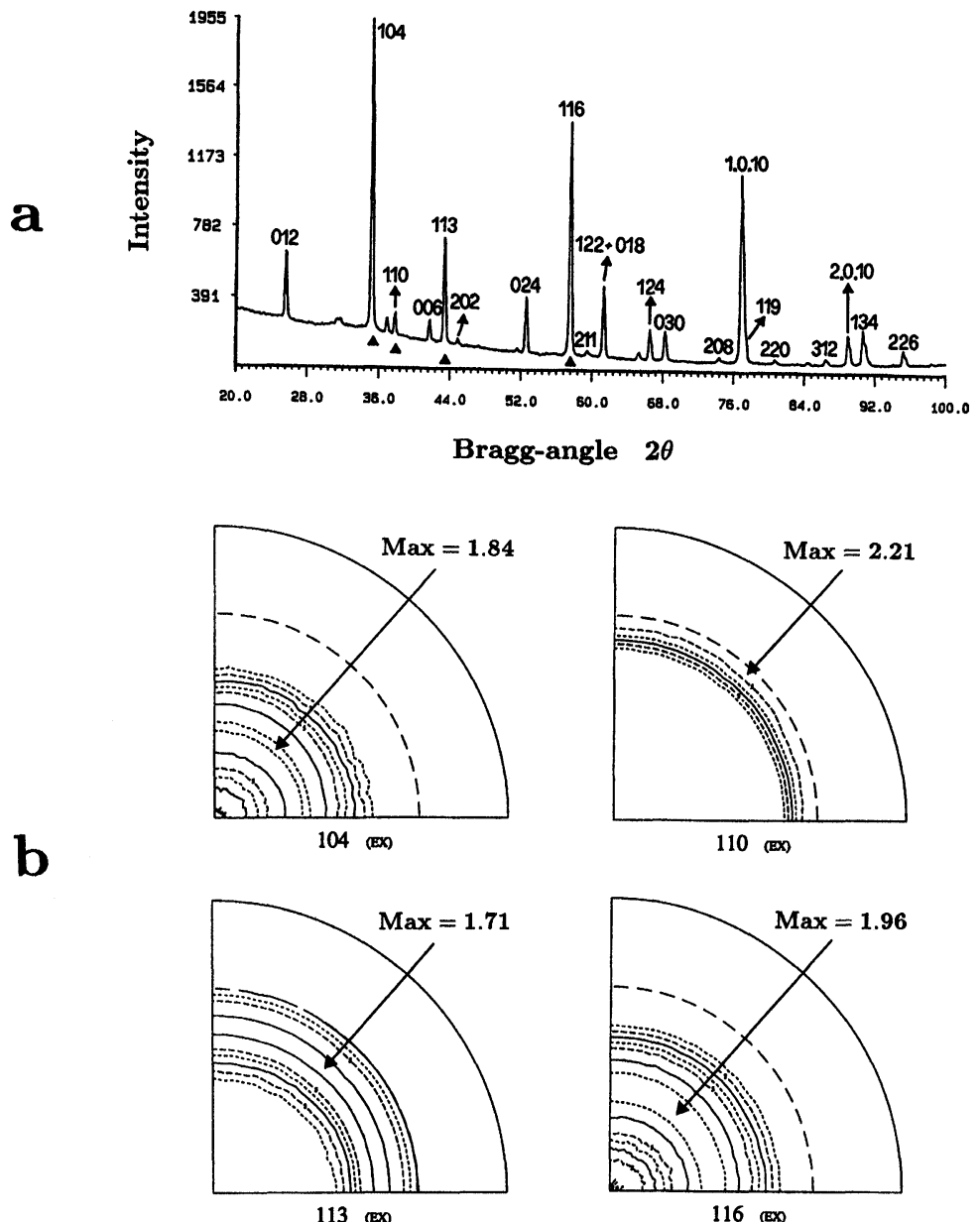

C
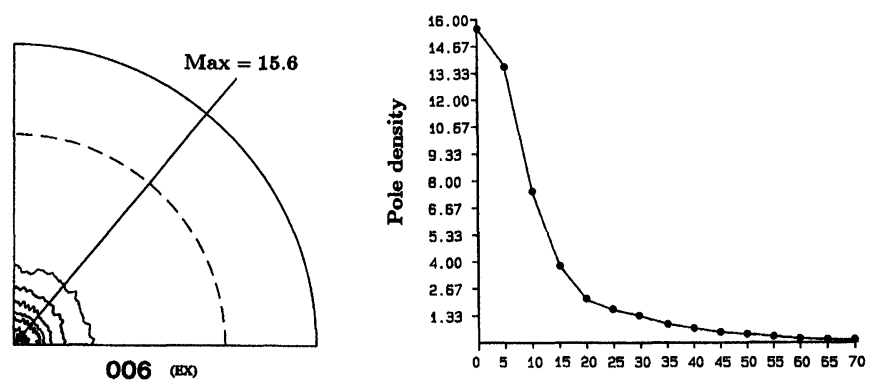

Sample tilt

Figure 3 Texture analysis in an $\mathrm{Al}_{2} \mathrm{O}_{3}$ substrate a) Diffraction diagram. b) Pole figures of some highintensity peaks. c) the (0006) pole figure. 
- The lower the symmetry the higher is the number of pole figures needed in order to reach a sufficient degree of series expansion, and that in turn is needed in order to reach sufficient angular resolving power in the ODF. As an example, 12 pole figures were used, for instance, in a monoclinic alloy compared with 3 as is usual in cubic metals.

- A second problem in low symmetry ODF-analysis is systematic peak coincidences (or near coincidences) which cannot even be resolved by the PSD-method mentioned above. This occurs, for instance, in $\mathrm{YBa}_{2} \mathrm{Cu}_{3} \mathrm{O}_{7-x}$ which has an orthorhombic structure but with very similar lattice parameters a and $b$. The same problem is also encountered in symmetries lower than orthorhombic if an angle of the unit cell is near $90^{\circ}$, e.g. $88.79^{\circ}$ in the above mentioned monoclinic case.

Summarizing these results, it can be said that texture analysis in the most important ceramic materials with lattice parameters in the range of $10 \AA$ or even up to $20 \AA$ are, today, possible with the new available techniques.

\section{Microabsorption}

In a two-phase material the intensity reflected in one phase may be absorbed only in the same phase or in both phases depending on particle size. In big particles the whole beam path is within the reflecting phase whereas with small particles, the beam passes both phases. If the particles have elongated forms, this may lead to anisotropic absorption as is illustrated in Figure 4. If the reflecting plane is parallel to the long axis the absorption coefficient is that of the reflecting phase, if it is perpendicular to the long axis then the average absorption coefficient of both phases is to be considered.
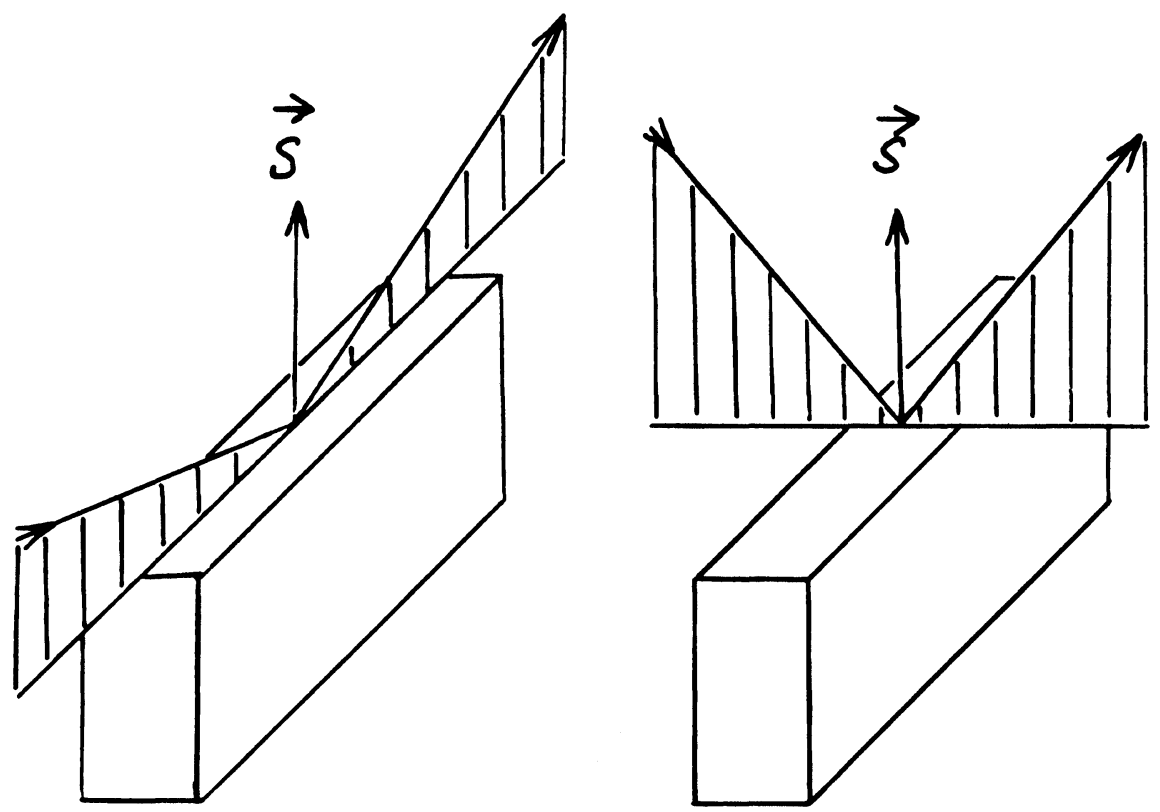

Figure 4 Schematic representation of aniostropic absorption as a consequence of the diffraction plane being parallel or perpendicular to the long axis of a particle. 


$$
\begin{aligned}
& \mu_{\|}=\mu_{1} \\
& \mu_{\perp}=\bar{\mu}=V_{1} \mu_{1}+V_{2} \mu_{2}
\end{aligned}
$$

where i designates the reflecting phase and $V_{i}$ are the volume fractions of the phases. In ceramics the effect may be particularly strong in case that one of the "phases" in eq(1) is air or even vacuum which has a very low absorption coefficient compared with that of the reflecting crystalline phase. Such voids may either be pores which have not been removed during sintering. Another kind of voids are microcracks for instance introduced by strongly anisotropic thermal expansion e.g. in $\mathrm{Al}_{2} \mathrm{TiO}_{3}$. According to the production process, shape and arrangement of such voids may be strongly anisotropic, thus leading to this effect. Also glass-phases, if they have another composition as the crystalline reflecting phase may give rise to anisotropic absorption.

\section{PARTICLE SHAPE TEXTURES}

Because of a higher anisotropy of surface and boundary energy, crystallites in ceramics often have crystallographic forms such as plates parallel to a crystallographic plane or needles parallel to a crystallographic direction. This has been used to determine textures in ceramics by microscopical methods. The texture is then defined according to the shape of a crystallite and not by the crystal lattice. These two definitions may give nearly the same result but they may also deviate strongly depending on the relationship between crystal shape and crystal lattice.

We assume that it is possible to fix a shape coordinate system $K_{s}$ in a crystallite according to clearly marked directions. As an example we may assume that the crystal has the shape of a brick and that it is possible to find the long, medium and short axes. The orientation of the coordinate system $K_{s}$ relative to the sample coordinate system $K_{A}$ is described by the rotation $g_{s}$ as is illustrated in Figure 5. The "shape texture" can then be defined in complete analogy to the definition of the crystallographic texture by the orientation distribution function

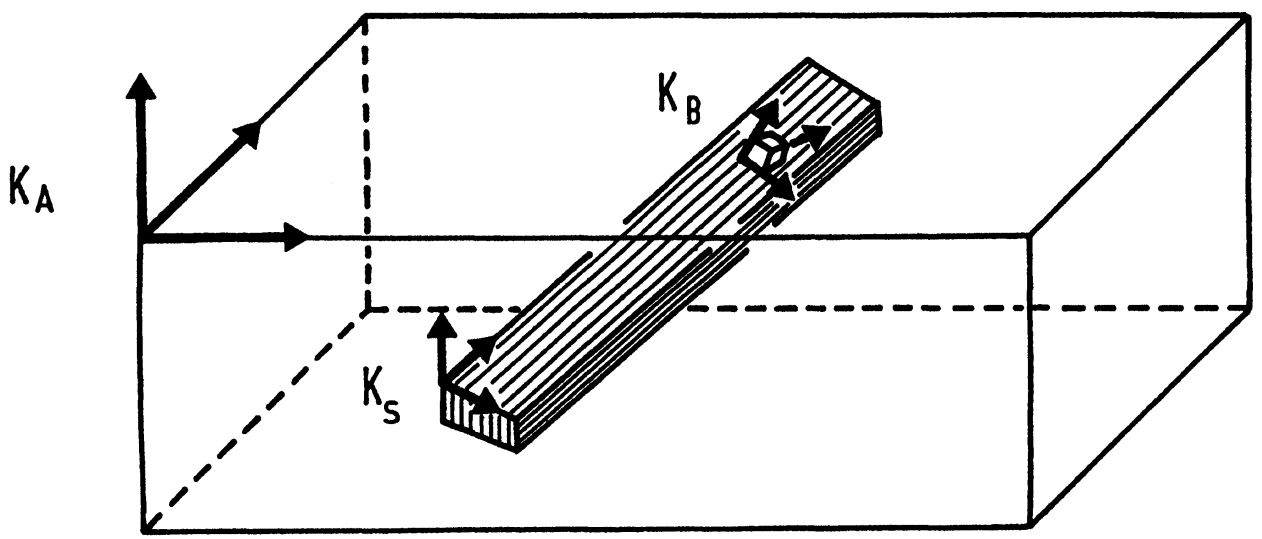

Figure 5 Definition of the sample coordinate system $K_{A}$, the shape coordinate system $K_{S}$ as well as the crystal corrdinate system $K_{B}$. 


$$
\frac{d V / V}{d g_{s}}=f^{s}\left(g_{s}\right) \quad ; K_{s}=g_{s} \cdot K_{A}
$$

The crystallographic texture is defined, as usual, by the crystal coordinate system $K_{B}$, also shown in Figure 5, and the orientation distribution function

$$
\frac{d V / V}{d g_{c}}=f^{c}\left(g_{c}\right) \quad ; K_{B}=g_{c} \cdot K_{A}
$$

In order to compare the two different definitions of texture we consider the orientation relation $\Delta g$ between crystallographic and shape coordinate system and its frequency distribution $w(\Delta g)$ - which may be called the "habitus function" of the crystalline particles

$$
\frac{d V / V}{d \Delta g}=w(\Delta g) \quad ; K_{s}=\Delta g \cdot K_{B} ; \quad g^{s}=\Delta g \cdot g^{c}
$$

The two differently defined textures are then related to each other by

$$
f^{s}\left(g_{s}\right)=\oint f^{c}\left(\Delta g^{-1} \cdot g_{s}\right) \cdot w(\Delta g) d \Delta g
$$

The habitus function $w(\Delta \mathrm{g})$ may be a "narrow" function in the vicinity of a particular orientation relation $\Delta g_{0}$ or it may show broad scattering about $\Delta g_{0}$ till to completely random distribution as is illustrated schematically in Figure 6 for a one-dimensional case. In ceramics, a sharp distribution is often observed whereas in metals random distribution is assumed. This is, however, rather a tendency than a strict distinction between metals and ceramics.

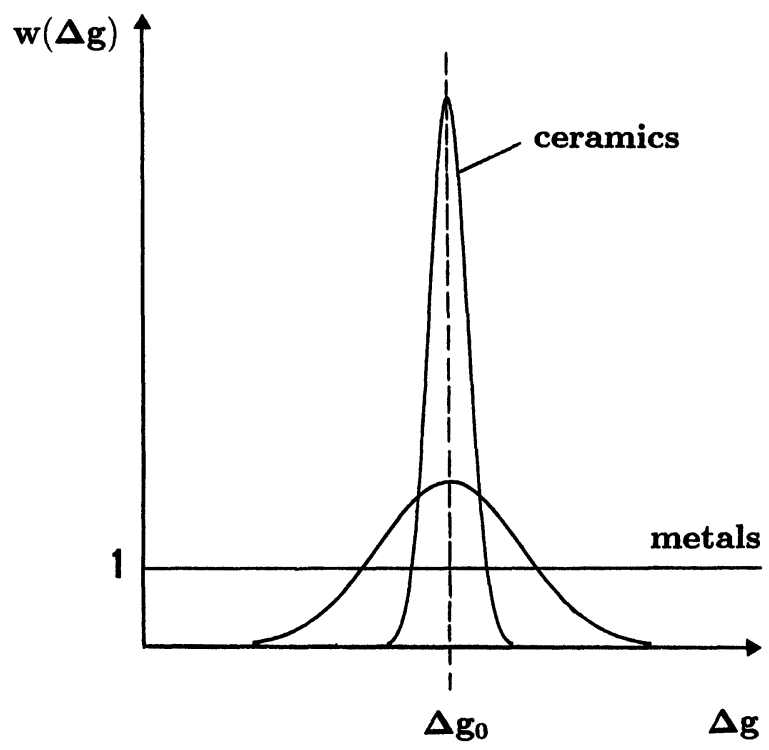

Figure 6 The relationship between crystal coordinate system $K_{B}$ and shape coordinate system $K_{S}$ described by the "habitus" function $w(\Delta g)$ (Schematic). 
Often it is not possible to fix a complete shape coordinate system $K_{s}$ but only one direction which may then be taken as the $\mathrm{X}_{3}$-direction of the system $K_{s}$. This is the case for needles where only the needle axis can be specified or for plates where only the plate normal direction can be seen. A rotation about this direction is then arbitrary. The disribution functions $f$ and $w$ are then independent of a rotation about this direction. If the rotations $g_{s}$ and $\Delta g$ are specified in terms of Eulerian angles it is

$$
\begin{array}{ll}
f^{s}\left(g_{s}\right)=f^{s}\left(\varphi_{1}, \phi\right) & ; g_{s}=\left\{\varphi_{1} \phi \varphi_{2}\right\} \\
w(\Delta g)=w\left(\varphi_{1}, \phi\right) & ; \Delta g=\left\{\varphi_{1} \phi \varphi_{2}\right\}
\end{array}
$$

The shape texture function $f^{s}$, in this case, is identical with the pole figure of the preferred axis and the habitus function $w(\Delta g)$ describes the distribution of the particle axis in terms of the crystallographic axes i.e. it is the inverse pole figure of the particle axis. If the distribution $w(\Delta g)$ is narrow then it may further be assumed that it depends only on $\phi . \mathrm{Eq}(7)$ is then further specified

$$
w(\Delta g)=w(\phi) \quad ; \Delta g=\left\{\varphi_{1} \phi \varphi_{2}\right\}
$$

If in eq (5) the crystallographic texture $f^{c}$ and the habitus function $w$ are known the shape texture $f^{s}$ can be calculated. The inverse problem, calculation of the crystallographic texture from the shape texture is also possible but only under certain conditions. It is not possible if the habitus function has the form eq(7) or eq(8). In this case a rotation of the crystallites about the preferred axis cannot be detected by shape texture measurements.

The three distribution functions can be expressed in terms of series expansions

$$
\begin{aligned}
& f^{c}\left(g_{c}\right)=\sum_{\lambda=0}^{L} \sum_{\mu=1}^{M(\lambda)} \sum_{v=1}^{N(\lambda)}{ }^{c} C_{\lambda}^{\mu v} \cdot \dot{T}_{\lambda}^{\mu v}\left(g_{c}\right) \\
& f^{s}\left(g_{s}\right)=\sum_{\lambda^{\prime}=0}^{L} \sum_{\mu^{\prime}=1}^{M^{\prime}\left(\lambda^{\prime}\right)} \sum_{v^{\prime}=1}^{N(\lambda)}{ }^{(\lambda)} C_{\chi}^{\mu^{\prime} v} \cdot \dot{\dot{T}}_{\chi}^{\mu^{\prime} v}\left(g_{s}\right) \\
& w(\Delta g)=\sum_{\lambda^{\prime \prime}=0}^{L} \sum_{\mu^{\prime \prime}=1}^{M^{\prime}\left(\lambda^{\prime \prime}\right)} \sum_{v^{\prime \prime}=1}^{\left.M \alpha^{\prime \prime}\right)}{ }^{\Delta} C_{\chi^{\prime \prime}}{ }^{\mu^{*} v^{\prime}} \cdot \dot{:}_{\chi^{*}}^{\mu^{*} v^{\prime \prime}}(\Delta g)
\end{aligned}
$$

Where $(\cdot)$ symbolizes the crystal symmetry, (:) symbolizes the sample symmetry and (:) symbolizes the shape symmetry. The quantities $M, M^{\prime}, N$ depend on these symmetries. The shape symmetry may be identical with crystal symmetry, it may be orthorhombic in case of three-axial ellipsoids and it may particularly be axial symmetry in case of rotational ellipsoids i.e. with only one preferred axis. In these cases it is

$$
\begin{aligned}
& =\mathrm{M}(\lambda) & & \text { crystal symmetry } \\
\mathrm{M}^{\prime}(\lambda) & =\mathrm{M}(\lambda)_{\text {orthorhombic }} & & \text { three-axial ellipsoid } \\
& =1 & & \text { rotation ellipsoid }
\end{aligned}
$$

Substituting the three series expansions in eq(5) one obtaines a relationship between the respective coefficients 


$$
{ }^{s} C_{\lambda}^{\sigma v}=\sum_{\mu=1}^{M(\lambda)} \frac{{ }^{\Delta} C_{\lambda}^{\sigma \mu} \cdot{ }^{c} C_{\lambda}^{\mu \nu}}{2 \lambda+1}
$$

This equation can be used from the right side to left to calculate the shape texture from the crystallographic texture and the habitus function. In the opposite direction it can be solved for the crystallographic texture coefficients with the shape coefficients given, only, if the number of $\sigma$-values is not smaller than that of the $\mu$-values, i.e. if the shape symmetry is the same as the crystal symmetry. Particularly, it cannot be solved if the shape symmetry is axial symmetry. The third way, eq(13) can be used, is to calculate the coefficients ${ }^{4} C_{\lambda}^{\sigma \mu}$ of the habitus function if the crystallographic texture and the shape texture are known. This requires that the sample symmetry is lower than the crystal symmetry.

\section{CONCLUSIONS}

Although the definition of texture is the same in all classes of materials and also the motivations for texture studies are, in principle the same, there are, nevertheless, some particular features that must be taken into consideration in texture studies in ceramiccs. These are mainly the more complex crystal structures giving rise to more complex, peak-rich diffraction diagrams.

Quantitative texture analysis in materials with such diagrams requires the measurement of whole diffraction spectra for each sample orientation followed by mathematical spectrum deconvolution of the overlapping peaks. This is particularly possible with position sensitive detectors. Hence, quantitative texture analysis, i.e. calculating the ODF from measured pole figures, is today possible for ceramic materials including those with low crystal symmetries and with lattice parameters in the range up to at least $10 \AA$ or even higher.

Texture-analysis in ceramics may, in some cases, be based on the shape of crystallites rather than on the crystal lattice. The "shape texture" is related to the "crystal lattice texture" by a "habitus"-function. This applies particularly to needle-shaped and platelike crystals.

\section{References}

Bowman, K. J. (1994). Textures in Ceramic Materials Proc. ICOTOM-10. Ed. H. J. Bunge, Materials Science Forum 157-162 43-56 Trans Tech Publ. Switzerland.

Bunge, H. J. (1991). Textures in Non-Metallic Materials Proc. ICOTOM-9 Textures and Microstructures 14-18, 283-322.

Bunge, H. J. (1993). Texture Analysis in Materials Science Second Ed. Cuvillier Verlag Göttingen.

Bunge, H. J. (Ed.) (1994). Proceedings of ICOTOM-10 Materials Science Forum 157-162 Trans Tech Publ. Switzerland.

Bunge, H. J., Siegesmund, S. Skrotzki, W. and Weber, K. (Eds.) (1994) Textures in Geological Materials DGM Informationsgesellschaft Oberursel.

Coulomb, P, (1972). Les textures dans les metaux de reseau cubic Dunod, Paris.

Dahms, M., Park, N. J. and Bunge H. J. (1994). Texture Analysis in the Monoclinic Martensitic Phase of a CuZnAl Shape Memory Alloy Proc. ICOTOM-10. Ed. H. J. Bunge Materials Science Forum 157-162, 507-514 Trans Tech Publ. Switzerland.

Hatherly, M. and Hutchinson, W. B. (1979). An Introduction to Textures in Metals The Institution of Metallurgists, London. 
Kudrjavzev, I. P. (1965). Textures in Metals and Alloys (russ.) Isdatelstwo Metallurgia Moscow. Park, N. J., Klein, H. and Dahlem-Klein, E. (1993). Program System ODF-Analysis for Cubic Crystal and Orthorhombic Sample Symmetry Ed. H. J. Bunge Cuvillier Verlag Göttingen.

Park, N. J., Klein, H. and Dahlem-Klein, E. (1995). Program System ODF-Analysis for Hexagonal, Tetragonal and Orthorhombic Symmetrie Ed. H. J. Bunge Cuvillier Verlag Göttingen (in preparation).

Sander, B. (1930). Gefügekunde der Gesteine Springer Verlag Berlin.

Underwood, F. A. (1961). Textures in Metal Sheets Macdonald, London.

Wassermann, G. and Grewen, J. (1962). Texturen metallischer Werkstoffe Springer Verlag Berlin. Wcislak, L., Bunge, H. J. and Nauer-Gerhardt, C. U. (1993). X-ray Diffraction Texture Analysis with a Position Sensitive Dectector Z. Metallkunde 84, 479-493.

Wcislak, L. and Nauer-Gerhardt, C. U. (1994). PSD-based Texture Investigations in Non-metallic Materials Z. Metallkunde 85, 564-573.

Wenk, H. R. (Ed.) (1985). Preferred Orientation in Deformed Metals and Rocks Academic Press, Orlando. 